\title{
Mobile Point Defects and Atomic Basis for Structural Transformations of a Crystal Surface
}

\section{Citation}

Hwang, I.-S., S. K. Theiss, and J. A. Golovchenko. 1994. "Mobile Point Defects and Atomic Basis for Structural Transformations of a Crystal Surface." Science 265 (5171) (July 22): 490-496. doi:10.1126/science.265.5171.490..

\section{Published Version}

doi:10.1126/science.265.5171.490

\section{Permanent link}

http://nrs.harvard.edu/urn-3:HUL.InstRepos:28488566

\section{Terms of Use}

This article was downloaded from Harvard University's DASH repository, and is made available under the terms and conditions applicable to Other Posted Material, as set forth at http:// nrs.harvard.edu/urn-3:HUL.InstRepos:dash.current.terms-of-use\#LAA

\section{Share Your Story}

The Harvard community has made this article openly available.

Please share how this access benefits you. Submit a story.

Accessibility 


\title{
Mobile Point Defects and Atomic Basis for Structural Transformations of a Crystal Surface
}

\author{
[Articles]
}

Hwang, Ing-Shouh*; Theiss, K Theiss; Golovchenko, J. A. **

I.-S. Hwang is with the Division of Applied Sciences, Harvard University, Cambridge, MA 02138, USA. S. K. Theiss is with the Department of Physics, Harvard University, Cambridge, MA 02138, USA. J. A. Golovchenko is with the Department of Physics and Division of Applied Sciences, Harvard University, Cambridge, MA 02138, and Rowland Institute for Science, Cambridge, MA 02142, USA.

* Present address: Institute of Physics, Academia Sinica, Taipei, Taiwan, Republic of China.

** To whom correspondence should be addressed.

\section{Abstract춰}

Structural transformations on elemental semiconductor suraces

typically occur above several hundred degrees Celsius, and the atomic motions involved are extremely rapid and difficult to observe. However, on the (111) surface of germanium, a few lead atoms catalyze atomic motions so that they can be observed with a tunneling microscope at temperatures below 80 degrees Celsius. Mass transport and structural changes are caused by the creation and propagation of both vacancylike and interstitial-like point defects within the crystal surface. The availability of dangling bonds on the surface is critical. A detailed atomic model for the observed motions has been developed and is used to explain the structural phase transition $\mathrm{Ge}(111)-\mathrm{c}(2$ times 8 ) double head arrow 1 times 1, which occurs near 300 degrees Celsius.

Interest in diffusion and phase transitions can be said to date back to the earliest days of metallurgy. That interest continues unabated today because our ability to create complex materials with useful properties and the stability of these materials depend on these phenomena. Both diffusion and structural transformations proceed through mechanisms that operate on the atomic scale, and fundamental understanding has come from indirect methods and reasoning. In single crystals, bulk diffusion is effected primarily by the motion of point defects: vacancies and interstitial atoms $[1,2]$. A vacancy can move from one 
substitutional site in a lattice to another and thus lead to mass transport in the opposite direction. An interstitial atom may either diffuse through the lattice until it combines with a vacancy (the interstitial mechanism), or it may replace a substitutional atom, displacing that atom into an adjacent interstitial site (the interstitialcy or kick-out mechanism). Diffusion that is constrained entirely within a single plane of atoms at a surface may be viewed as the twodimensional analog of bulk diffusion. It likewise requires mobile point defects. Unlike the bulk, however, the surface is accessible to direct, atomic-scale study. In addition, improved understanding of surface dynamics is of importance in its own right and would benefit disciplines extending from chemistry to electronics.

In recent years, there has been growing interest in the use of the scanning tunneling microscope (STM) for real time and space observations of the fundamental mechanisms of surface mass transport [3-8]. One of the main problems in such a study is the slow scanning speed of the current generation of tunneling microscopes, which often cannot follow atomic motions occurring on crystal surfaces. A possible solution is to design STMs that can somehow take images more quickly. Another is to find a way to catalyze the atomic motions such that they occur at lower temperatures, where atomic motions are slower and can be clearly identified by the STM. Such catalysis could be of great practical use as well, for example, in crystal growth.

A small number of impurity $\mathrm{Pb}$ atoms on a Ge surface catalyze atomic motions and structural changes without altering the basic mechanisms involved. The present study evolved from our work on $\mathrm{Pb}$ atom diffusion, which involves interchanges of individual $\mathrm{Pb}$ atoms with $\mathrm{Ge}$ adatoms [4]. The diffusion tends to occur along the Ge adatom row direction, and about half of the interchanges are long jumps (movements of more than one atomic spacing). Additionally, a large number of adjacent $\mathrm{Ge}$ adatoms in a row in the $\mathrm{c}(2$ times 8 ) surface reconstruction can shift in position concertedly along the row direction like beads on an abacus [5]. We have now identified the basic mechanism, which involves mobile point defects, that engenders both surface diffusion and the rich collection of structural transformations the Ge surface harbors.

Here we present STM observations of a lightly Pb-doped Ge(111) surface from 25 degrees to 80 degrees Celsius and of a pure Ge(111) surface from 25 degrees to 220 degrees Celsius. We identify two mobile point defects. One consists of a "partially interstitial" atom in 
the $c$ (2 times 8 ) reconstruction. The other complementary point defect consists of a "partial vacancy" that can alternatively be viewed as a localized high density of surface dangling bonds. The propagation of these two types of mobile point defects is responsible for the structural changes (and mass transport) observed on the Pb-doped $\mathrm{Ge}(111$ ) surface, and their prolific creation at high temperatures leads to the structural transition $\mathrm{Ge}(111)$-c(2 times 8$)$ double head arrow 1 times 1. Adding a small number of $\mathrm{Pb}$ atoms to the $\mathrm{Ge}(111)$ surface reduces the activation energy of formation for these two types of mobile point defects and makes their study possible.

\section{Experimental Method 4}

Several features make the lightly Pb-doped Ge(111) surface a favorable one for the STM study of detailed atomic motions. Pb and $\mathrm{Ge}$ have negligible mutual bulk solubility at all temperatures [9], and $\mathrm{Pb}$ atoms do not evaporate from the surface below 300 degrees Celsius [10]. Therefore, $\mathrm{Pb}$ atoms remain at the surface after deposition.

Furthermore, $\mathrm{Pb}$ atoms are easily distinguished from $\mathrm{Ge}$ adatoms by the STM and thus can serve as markers, which allow determination of the registry and motion of adatoms.

Our experiments were performed with a homemade STM in an ultrahigh-vacuum chamber with base pressure of 6 times $10^{-11}$ torr. Samples of 1- to 5-ohm middot cm n-type Ge(111) were used. Clean $\mathrm{Ge}(111)-\mathrm{c}(2$ times 8$)$ surfaces were prepared by successive Ne ion sputtering and annealing until very sharp c(2 times 8 ) low-energy electron diffraction (LEED) spots were obtained from the entire sample. These surfaces were then either used to study the initial stage of the $\mathrm{c}(2$ times 8$)$ double head arrow 1 times 1 phase transition on clean $\mathrm{Ge}(111)$ or were used as a substrate for $\mathrm{Pb}$ deposition. On the latter surfaces, about 0.02 monolayer (one monolayer consists of one $\mathrm{Pb}$ atom per first layer $\mathrm{Ge}$ atom, a concentration of 7.22 times $10^{14}$ $\mathrm{cm}^{\text {negative }} 2$ ) of $\mathrm{Pb}$ was deposited from an effusion cell with system pressure below 4 times $10^{\text {negative }} 10$ torr. The sample was then transferred to the STM stage. The sample temperature was varied by resistive heating during the experiment. I mages were taken between 25 degrees and 80 degrees Celsius for the $\mathrm{Pb}$-doped surfaces and up to similar to 200 degrees Celsius for the clean surface. Before taking STM images at a new temperature, we waited 30 min to 3 hours to allow the instrument to stabilize thermally. Typically, the STM was operated at a constant current of 20 to $50 \mathrm{pA}$ and with a bias on the sample of about positive $2 \mathrm{~V}$. 


\section{Ge(111)-c(2 times 8) Reconstruction and Adatom Motions =1}

An STM image of a clean $\mathrm{Ge}(111)$ surface at room temperature is shown in Figure 1 . Most of the region has the $c(2$ times 8$)$ reconstruction ( $a$ unit cell is labeled $A$ ). Imperfections in the reconstruction, such as extra adatom rows that result in three rows each of 2 times 2 (at B) and c( 2 times 4 ) (at C), can be seen. There is also a small domain of $\mathrm{c}$ ( 2 times 4 ) rotated relative to the large domain of $\mathrm{c}(2$ times 8 ) (labeled D).

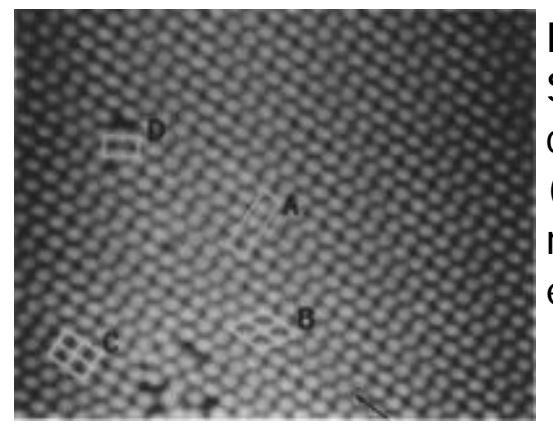

Figure 1. A 210 angstrom by 210 angstrom STM image of a clean $\mathrm{Ge}(111)$ surface. Unit cells of (A) $c(2$ times 8$)$, (B) 2 times 2 , and (C) $\mathrm{c}(2$ times 4$)$ and (D) a defect near a rotated region of c(2 times 4$)$ are marked. An extra adatom row is indicated with an arrow.

In a perfect $\mathrm{Ge}(111)-\mathrm{c}(2$ times 8 ) surface reconstruction Figure 2, the Ge adatoms occupy the so-called "top, four-coordinated" sites, or $\mathrm{T}_{4}$ sites, on the bulk terminated substrate [11-15]. Each adatom in a $T_{4}$ site forms three bonds to the first layer rest atoms (small open circles in Figure 2 [it also interacts with the atom directly below it, so this site is considered four-coordinated Figure 3]. The rest atom dangling bonds (RADaBs) (small filled circles in Figure 2 have the same relative configuration as do the adatoms. A perfect c(2 times 8 ) structure has a stacking order along the surface in which rows of 2 times 2 and c( 2 times 4$)$ subunit cells alternate. The adatom rows are along one of three equivalent $<01$ bar $1>$ substrate directions; thus, there are three possible differently oriented $c(2$ times 8$)$ domains, rotated 120 degrees from each other, that break the threefold symmetry of the substrate. We will refer to the row direction as [01 bar 1] Figure 2. 


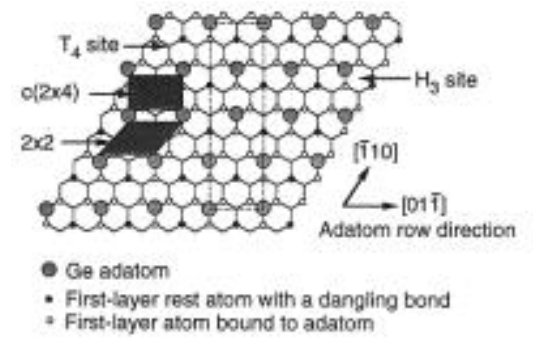

Figure 2. Simple adatom model of Ge(111)c(2 times 8). This reconstruction breaks the threefold symmetry of the bulk. The adatom row direction is defined as [01 bar 1]. A unit cell is indicated by dashed lines.

ayer rest atom with a danging bond

[Help with image viewing]

[Email umpstart To I mage]

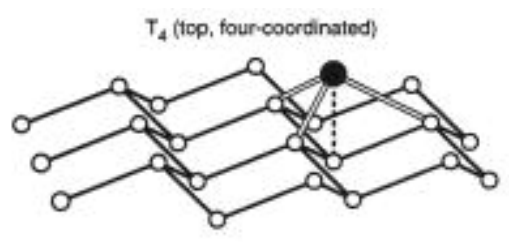

Figure 3. Model of an adatom movement from a $\mathrm{T}_{4}$ site to a metastable $\mathrm{H}_{3}$ site.
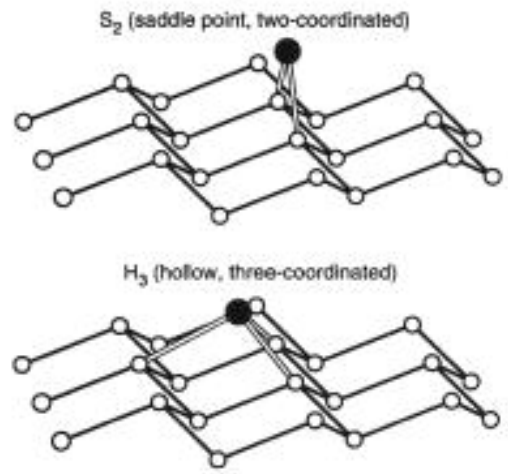

[Help with image viewing]

[Email Jumpstart To I mage]

On the clean Ge(111)-c(2 times 8) surface, errors in the stacking order can lead to extra adatom rows and result in three (or occasionally four) rows of 2 times 2 [11,16]. Three adatom rows forming a local $\mathrm{c}(2$ times 4$)$ arrangement are also seen, but not as often as extra rows of 2 times 2 . Examination of the model Figure 2 reveals that a $\mathrm{c}(2$ times 4$)$ subunit cell can be converted into a 2 times 2 subunit cell by shifting all of the atoms on one row to the next $\mathrm{T}_{4}$ site in the [01 bar 1] direction. Such adatom row shifts have been 
observed on the Pb over $\mathrm{Ge}(111)$ surface [5]. They preserve the $2 \mathrm{x}$ periodicity along the row direction, and we can generally define structures that result from a series of row shifts as " $2 x$ row structures," or "row structures" in brief. The c( 2 times 8$), 2$ times 2 , and c(2 times 4$)$ arrangements are special cases of row structures.

An adatom in a "hollow, three-coordinated" site, or $\mathrm{H}\left({ }_{3}\right)$ site Figure 2, forms three covalent bonds with the substrate as it does on the $T_{4}$ site. It has been suggested by Aarts et al. [17] that a Ge adatom can move from a $\mathrm{T}_{4}$ site to a neighboring $\mathrm{H}_{3}$ site and vice versa by switching only one bond with the rest atoms Figure 3. The $\mathrm{T}_{4}$ right arrow $\mathrm{H}_{3}$ right arrow $\mathrm{T}_{4}$ shift is the fundamental mechanism in our model of $\mathrm{Ge}$ adatom motions. Recently, first-principles molecular dynamics simulations of the $\mathrm{Ge}(111)-\mathrm{c}(2$ times 8$)$ surface have shown that the energy barrier is $0.8 \mathrm{eV}$ for a Ge adatom to hop from its $\mathrm{T}\left({ }_{4}\right)$ site to a neighboring $\mathrm{H}_{3}$ site and $0.2 \mathrm{eV}$ for it to hop back to the original $\mathrm{T}_{4}$ site [18]. With the Arrhenius equation Equation 1 where $t$ is the mean lifetime, $E_{D}$ is the activation energy, $v$ is the attempt frequency (similar to $10^{13} \mathrm{~S}^{\text {negative }} 1$ ), $\mathrm{k}_{\mathrm{B}}$ is the Boltzmann constant, and $\mathrm{T}$ is the temperature--we can estimate the mean lifetime for a Ge adatom to stay at the $T_{4}$ site to be several seconds and that on an $\mathrm{H}_{3}$ site to be $10^{\text {negative }} 10 \mathrm{~s}$ at room temperature. Because the latter lifetime is 10 to 11 orders of magnitude shorter than the former one, structural changes are very unlikely to occur in the $\mathrm{Ge}(111)-\mathrm{c}(2$ times 8$)$ reconstruction at room temperature. However, they can occur at high temperatures, at which $\mathrm{Ge}$ adatoms have a much higher hopping rate to the $\mathrm{H}_{3}$ site (the hopping rate is the inverse of the lifetime on the $T_{4}$ site.)

$t=v^{-1} \exp \left(E_{\mathrm{D}} / k_{\mathrm{B}} T\right)$

Equation 1

[Help with image viewing]

[Email umpstart To I mage]

The motion of a $\mathrm{Ge}$ adatom to an $\mathrm{H}_{3}$ site requires the presence of a RADaB there. If no RADaB is available, the adatom will be unable to switch its bond and the motion is very unlikely to occur (throughout the rest of this article, if Ge motions occur, we assume that the RADaBs are available without always mentioning it specifically). The value of $E_{D}$ for an adatom to hop from its $\mathrm{T}_{4}$ site to a neighboring $\mathrm{H}_{3}$ site varies with the surrounding adatom and RADaB configuration. 
Motions involving the simultaneous breaking of two or more Ge-Ge bonds are not considered here because of their high activation energies relative to the highest temperature discussed here (350 degrees Celsius) [19].

\section{Structural Excitations in the Lightly Pb-Doped Ge(111) Surfaceㄹㅊ}

When a small quantity of $\mathrm{Pb}$ is deposited on $\mathrm{Ge}(111)$, most of the $\mathrm{Pb}$ atoms replace $\mathrm{Ge}$ adatoms in the $\mathrm{c}(2$ times 8$)$ reconstruction after deposition [4]. We call these "substitutional" Pb atoms. A Pb-Ge bond is much weaker than a Ge-Ge bond [5-10]. It has been suggested that at room temperature, the substitutional $\mathrm{Pb}$ atoms hop rapidly (greater than $10^{3} \mathrm{~S}^{\text {negative }} 1$ ) between their $\mathrm{T}\left({ }_{4}\right)$ sites and the neighboring $\mathrm{H}_{3}$ sites, with a lifetime on the $T_{4}$ sites at least an order of magnitude longer than that on the $\mathrm{H}_{3}$ sites [5]. In contrast, Ge atoms hop to the $\mathrm{H}_{3}$ site at a rate of about $0.1 \mathrm{~s}^{\text {negative }} 1$, with a lifetime 10 to 11 orders of magnitude longer than that on $\mathrm{T}_{4}$. Metastable structures with $\mathrm{Pb}$ atoms trapped on the $\mathrm{H}_{3}$ site were occasionally observed, and they can last minutes before relaxation near room temperature [5]. This result suggests that the energy difference between occupying $\mathrm{T}_{4}$ and $\mathrm{H}_{3}$ sites is much smaller for $\mathrm{Pb}$ than for $\mathrm{Ge}$ adatoms. Because of the weakness of the $\mathrm{Pb}-\mathrm{Ge}$ bond relative to the covalent $\mathrm{Ge}-\mathrm{Ge}$ bond and the much longer $\mathrm{H}_{3}$ site lifetime of $\mathrm{Pb}$ atoms compared with $\mathrm{Ge}$ adatoms, structural changes of $\mathrm{Ge}$ adatoms in the lightly $\mathrm{Pb}$-doped surface occur at lower temperatures than in the pure Ge(111) surface.

\section{Adatom row shifts}

On the lightly $\mathrm{Pb}$-doped $\mathrm{Ge}(111)$ - $\mathrm{c}(2$ times 8$)$ surface, the Ge adatoms in the same row can shift in concert along the row by one primitive vector of the substrate and trap a $\mathrm{Pb}$ atom on a surface interstitial site $\left(\mathrm{H}_{3}\right)$. Some time later, the structure can relax [5]. We present a detailed mechanism for the creation and annihilation of this metastable structural excitation on the basis of our new STM observations.

An example of the $\mathrm{c}(2$ times 8 ) structure with a substitutional $\mathrm{Pb}$ atom is shown schematically in Figure $4 \mathrm{~A}$. The $\mathrm{Pb}$ atom hops rapidly between the $\mathrm{T}_{4}$ site and neighboring metastable $\mathrm{H}_{3}$ sites near room temperature. The Ge adatoms also hop in a similar way but at a much slower rate because of higher activation energies. While the $\mathrm{Pb}$ atom has hopped to the left $\mathrm{H}_{3}$ site, there is a chance that Ge adatom 1 will hop left to a $\mathrm{T}_{4}$ site through a metastable $\mathrm{H}_{3}$ site Figure 4B. The $\mathrm{Pb}$ atom is then trapped on the $\mathrm{H}_{3}$ site because there are no RADaBs 
available at any neighboring site. Adatom 2 may also move left through an $\mathrm{H}_{3}$ site to a new $\mathrm{T}_{4}$ site Figure 4C because several RADaBs to its left are available for bond switching during the adatom motion. The process can proceed, and adatom 3, adatom 4, and so forth move left one by one in the same manner as adatoms 1 and 2 . The result is a metastable structure like Figure 4D, which has been observed previously [5]. We call the above process a "vacancy-mediated" row shift because it features the propagation of a partial vacancy along the adatom row. It is analogous to vacancy diffusion in bulk material. The propagation normally ends at a domain boundary or a preexisting point defect.

The vacancy is "partial" because it is smaller than that which would be created by removing one adatom, as can be seen by inspection of Figure 4. Likewise, the $\mathrm{Pb}$ adatom trapped on an $\mathrm{H}_{3}$ site may be viewed as a "partially interstitial" atom. To limit cumbersome notation, we will refer to this simply as an "interstitial $\mathrm{H}_{3}$ site" atom.

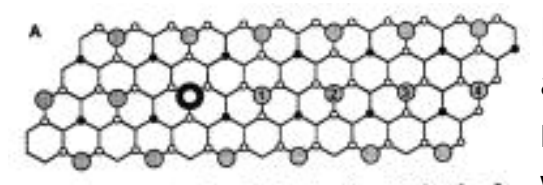

Figure 4. Model of a vacancy-mediated adatom row shift. (A) Schematic representation of a $\mathrm{C}(2$ times 8$)$ structure

$\cdot$

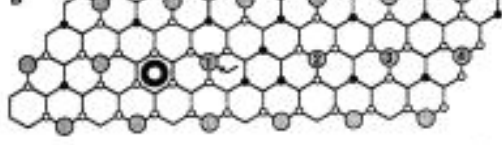

c
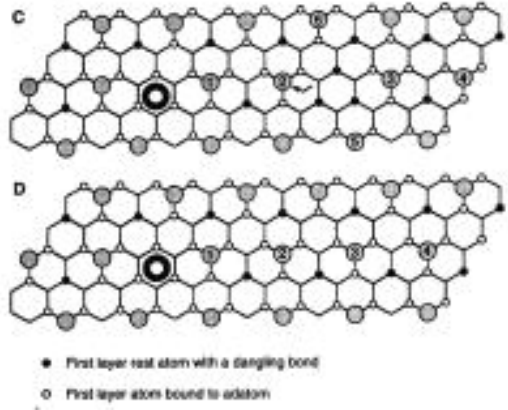

[Help with image viewing]

[Email umpstart To I mage] with a substantial $\mathrm{Pb}$ atom. ( $\mathrm{B}$ and $\mathrm{C}$ )

Intermediate steps in adatom row shift, assuming only that atoms move to nearestneighbor sites with available RADaBs. (D) Final observed, metastable structure.

The metastable structure in Figure 4D has been observed to relax back to that in Figure 4Aafter a period of time. The relaxation is interpreted as another type of adatom row shift, which we call an "interstitial-site- 
mediated" row shift. For the structure in Figure 4D, the interstitial $\mathrm{H}_{3}$ site $\mathrm{Pb}$ atom can move back to its original substitutional $\mathrm{T}_{4}$ site while $\mathrm{Ge}$ adatom 1 hops right to an $\mathrm{H}_{3}$ site Figure 5A. Adatom 1 is then trapped on the $\mathrm{H}_{3}$ site. It has a chance to move right to a $\mathrm{T}_{4}$ site if adatom 2 hops right to an $\mathrm{H}_{3}$ site Figure 5B. In a similar manner, the trapped adatom 2 can move right to $\mathrm{T}_{4}$ site if adatom 3 hops right to an $\mathrm{H}_{3}$ site Figure $5 \mathrm{C}$. The process can continue until the structure relaxes back to the original structure Figure 5D. This adatom row shift is characterized by the propagation of an interstitial $\mathrm{H}_{3}$ site as new Ge adatoms are sequentially trapped. It is similar to a series of the interstitialcy or kick-out mechanisms in bulk material.

Direct observation of a partial vacancy or of an interstitial $\mathrm{H}_{3}$ site $\mathrm{Ge}$ atom would be strong evidence for the mechanisms proposed above. However, the small size of the point defects, their low concentration, and their high mobility make such observations very difficult.

We observed the propagation of an interstitial $\mathrm{H}_{3}$ site $\mathrm{Ge}$ atom along the row by carefully tuning the data acquisition conditions. Two consecutive 70 angstrom by 75 angstrom STM images, taken 51 seconds apart at similar to 70 degrees Celsius are shown in Figure 6. In Figure $6 \mathrm{~A}$, an adatom row shift is occurring in the row indicated by the arrow below the image. Some atoms appear as incomplete circles because of $\mathrm{Ge}$ adatom motion during data acquisition; that is, the atom is in one position when we start to scan it and moves to a new position before we finish. In the same row, below the "incomplete" atoms, is an interstitial $\mathrm{H}_{3 \text {-site }} \mathrm{Ge}$ adatom like adatom 3 in Figure $5 \mathrm{C}$. In Figure 6B, the interstitial $\mathrm{H}_{3}$ site propagates along the row. An interstitial-site-mediated row shift is occurring. Each Ge adatom stays trapped on the interstitial $\mathrm{H}_{3}$ site for only seconds or less before the structure propagates. The adatom structure on one side of the interstitial $\mathrm{H}_{3}$ site $\mathrm{Ge}$ adatom is in 2 times 2 local order, and that on the other side is in c(2 times 4$)$ local order, exactly as was observed previously around an interstitial $\mathrm{H}_{3}$ ) site $\mathrm{Pb}$ adatom [5]. 

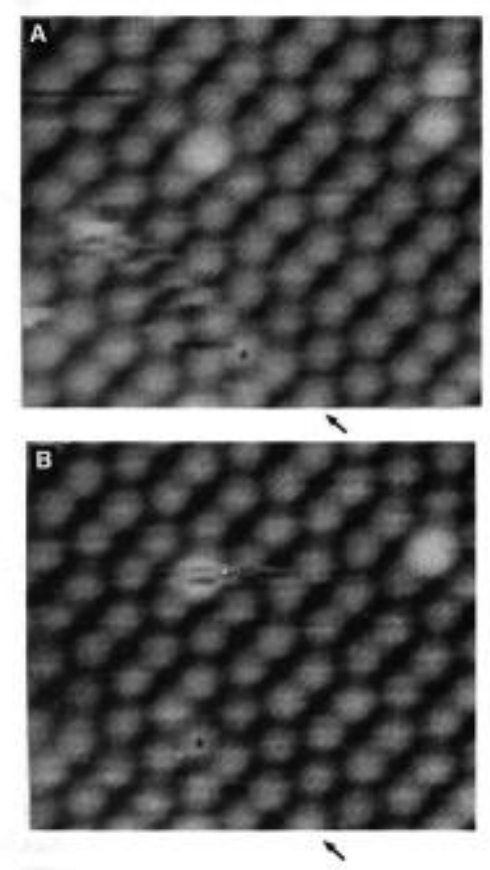

[Help with image viewing]

[Email Jumpstart To Image]
Figure 6 . Two consecutive 70 angstrom by 75 angstrom STM images of an interstitial-sitemediated row shift occurring in the row labeled with an arrow. Sample at similar to 70 degrees Celsius, images taken at $(A) t$ equals $0 \mathrm{~s}$ and $(B) \mathrm{t}$ equals $51 \mathrm{~s}$. Dark and bright atoms are $\mathrm{Ge}$ and $\mathrm{Pb}$ atoms, respectively. The Ge adatoms trapped on an $\mathrm{H}_{3}$ site are marked with an asterisk.

Although row shifts were seen in about 100 of our STM images, interstitial $\mathrm{H}_{3}$ site $\mathrm{Ge}$ atoms were only directly observed in about 10 of the more than 1000 images analyzed. They were always accompanied by evidence of row shifts ("incomplete" atoms) in subsequent images. The characteristic local structure of a vacancy-mediated row shift Figure 4B or Figure 4C was not observed, but this complementary process is required to explain atomic motions observed in this system. A partial vacancy probably migrates much faster than an interstitial $\mathrm{H}_{3}$ site, so a vacancy-mediated row shift always appears in STM images as a chain of $\mathrm{Ge}$ adatoms moving along the row direction simultaneously. This result is not surprising because vacancies and interstitial atoms also have different diffusion coefficients in the bulk [2].

For clarity, the propagation of a partial vacancy and an interstitial $\mathrm{H}_{3}$ site Ge adatom in Figure 4 and Figure 5 were drawn in one direction only. Actually, they can propagate back and forth along the adatom row, and STM images such as the "incomplete" Ge adatoms seen in Figure 6 Aindicate this back and forth migration. 
A

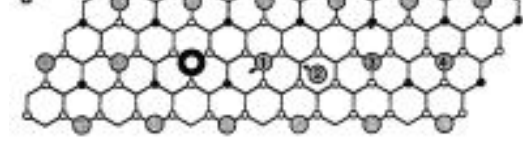

c

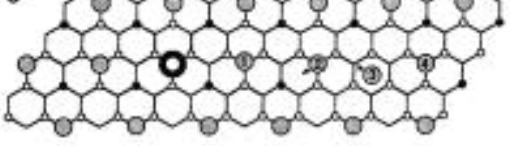

D

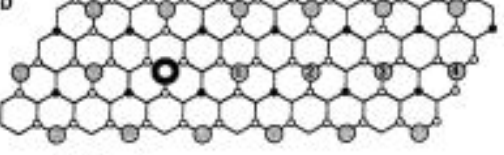

O An actatan

( $p$ siom

[Help with image viewing]

[Email Jumpstart To Image]
Figure 5. Model of an interstitial site-

mediated adatom row shift. (A through $C$ )

Intermediate steps in adatom row shift, assuming only that atoms move to nearestneighbor sites with available RADaBs. (D) Original, observed structure.

\section{Nucleation of the metastable, rotated row structure.춰}

Although most atomic motions in the $\mathrm{c}(2$ times 8$)$ reconstruction occur along the adatom rows, we also observed metastable excitations that break the row structure locally Figure 7 . A small domain of the 2 times row structure formed with its adatom rows running in a different $<01$ bar $1>$ direction from the original one. It formed within a single large region of $c(2$ times 8$)$ and disappeared again in the following image. The arrangement can be explained as follows. Consider again the case of Figure 4C. Adatom 5 (or adatom 6) can hop into the partial vacancy before adatom 3 moves, thus stopping the row motion. The hop also leaves behind a partial vacancy, which allows further motions of surrounding adatoms. The partial vacancy propagates downward and then moves away along another adatom row (indicated by the arrows in Figure 7. Because the effect of the two types of defects is complementary, this structure could also be formed by an interstitialtype row shift entering on the lower row, turning upward, and then propagating away along the upper row. At the boundary between the new and the original domains of the row structure, there are local structures with two neighboring RADaBs and with three Ge adatoms in the square root of 3 times square root of 3 configuration. These local 
structures are commonly observed at domain boundaries of c( 2 times 8).

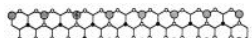

0.050000

0015000505

000002002025

$\therefore$

[Help with image

viewing]

[Email ] umpstart

To Imagel
Figure 7. Schematic representation of an excitation that broke the row structure locally. Atom numbers correspond to those in Figure 4C. Coarsely dashed lines show the row direction of the new structure; finely dashed lines mark regions of local square root of 3 times square root of 3 order. The arrows indicate the motions required to convert to this structure from the one in Figure 4C.

The formation of a rotated domain of the row structure inside a large, perfect domain of c(2 times 8 ) was observed only twice out of more than 1000 images. The surface relaxes back to the original row structure after a period of time. The rarity of this second kind of metastable excitation relative to the adatom row motions indicates that it is more energetically favorable for adatom 3 in Figure 4Cto move than it is for adatom 5 , even though both make a similar $\mathrm{T}_{4}$ right arrow $\mathrm{H}_{3}$ right arrow $\mathrm{T}_{4}$ motion. Essentially, a mobile point defect has a lower activation energy to propagate along the adatom row than away from it. Out-of-row motion becomes more probable at high temperature, however, and we believe that this is the mechanism by which new domains of the row structure can nucleate within preexisting domains, leading to the loss of long-range adatom order at high temperatures on the pure Ge(111) surface.

A small, rotated domain of the row structure is more likely to form near defects on the Ge(111) surface than inside a perfect domain of $\mathrm{c}(2$ times 8 ). For example, there are immobile defects that appear to be dark in unoccupied-state tunneling images but bright in the occupied-state images. These defects are like those observed by Klitsner et al., who found that areas on the Ge(111) surface that had reacted with oxygen appeared dark in unoccupied-state images but bright in occupied-state images [20]. We conclude that the defects are caused by reactions with residual gas atoms from the vacuum chamber that passivate a few RADaBs. There are three reasons: (i) They are similar to the defects caused by oxidation; (ii) their density is 
found to increase with time; and (iii) they are immobile near room temperature (they can move at high temperatures but are still much less mobile than Ge adatoms). The Ge adatoms move around these defects rather than occupy them because RADaBs are no longer available there. Thus, the immobile defects can deflect the mobile point defects out of their original row, giving rise to the rotated row structure.

Three consecutive tunneling images of structural changes occurring around an immobile defect at 60 degrees Celsius are shown in Figure 8. The atomic positions can be determined unambiguously Figure 9. Because the defect is not mobile, it can be used as a reference for the motions. Between Figure $9 \mathrm{~A}$ and Figure $9 \mathrm{~B}$, the $\mathrm{Pb}$ atom marked with $\mathrm{a}$ "middot" moves down from an $\mathrm{H}_{3}$ site to another $\mathrm{H}_{3}$ site (a long jump) and a Ge adatom moves up from a $\mathrm{T}_{4}$ site to another $\mathrm{T}_{4}$ site through the $\mathrm{H}_{3}$ site in between. The $\mathrm{Pb}$ atom marked with an " $\mathrm{x}$ " also moves into the image through a long jump and occupies an $\mathrm{H}_{3}$ site. This motion is accompanied by a series of $\mathrm{Ge}$ adatom motions like those shown in Figure 7. The motion of these $\mathrm{Ge}$ adatoms traps the $\mathrm{Pb}$ atom marked with a "--" on an $\mathrm{H}_{3}$ site. Between Figure 9B and Figure 9C, $\mathrm{Pb}$ atom " $x$ " leaves this region through another long jump and $\mathrm{Pb}$ atom "- " makes a long jump down to a $\mathrm{T}_{4}$ site. A series of $\mathrm{Ge}$ atom movements occurs either because of the propagation of the partial vacancy created by the departure of $\mathrm{Pb}$ atom " $\mathrm{x}$ " or, alternatively, because of the propagation of an interstitial $\mathrm{H}_{3}$ site from $\mathrm{Pb}$ atom "--." 


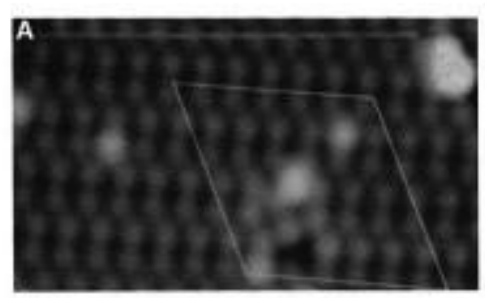

Figure 8. Three consecutive 115 angstrom by 70 angstrom STM images of structural changes, taken at (A) t equals $0 \mathrm{~s},(B) 82 \mathrm{~s}$, and (C) $167 \mathrm{~s}$. The regions inside the solid lines are analyzed in Figure 9.
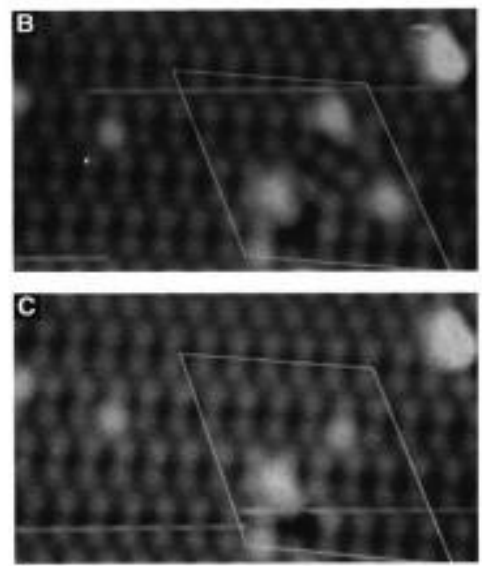

[Help with image viewing] [Email ] umpstart To I mage] 


\section{A}

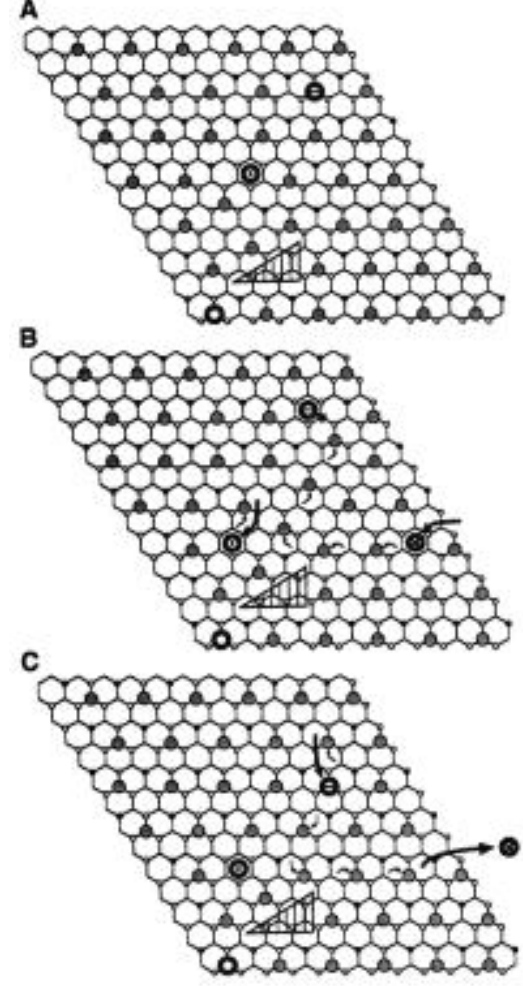

[Help with image viewing]

[Email umpstart To I mage]
Figure 9. Model for structural changes occurring in Figure 8. The arrows are drawn assuming only that Ge adatoms hopped to nearest-neighbor sites with available RADaBs. As in Figure 8 , the models correspond to $(A) t$ equals $0 \mathrm{~s},(\mathrm{~B}) 82 \mathrm{~s}$, and (C) $167 \mathrm{~s}$.

\section{Energetics on the Pure Ge(111) Surface t}

We can compare the energetics of various structures on $\mathrm{Ge}(111)$ by analyzing their frequency of occurrence in our tunneling images. In order of decreasing stability, these structures are c( 2 times 8$)$, other row structures, adatoms in square root of 3 times square root of 3 configurations or RADaBs on two neighboring first-layer atoms, and finally, interstitial $\mathrm{H}_{3}$ site atoms and partial vacancies. The $\mathrm{c}(2$ times 8) reconstruction is the ground state structure on $\mathrm{Ge}(111)$, and most of the area we see is in this configuration. However, as seen in Figure 1, extra adatom rows are occasionally seen on the Ge(111) surface at room temperature and result in three rows in local 2 times 2 or c( 2 times 4$)$ order. Three rows of 2 times 2 must be more stable than three rows of $\mathrm{c}(2$ times 4$)$ because the former structure is much more abundant in our tunneling images than the latter. Large domains of the row structure are more stable than small ones. At the boundaries between differently rotated domains of the row structure, we see local structures with three $\mathrm{Ge}$ adatoms in a square root of 3 times square root of 3 configuration or with two neighboring RADaBs. These 
structures exist only at domain boundaries, and thus, it can be concluded that they are not very favorable compared to the row structures. Even at the domain boundaries, structures with more than three square root of 3 times square root of 3 adatoms are rarely seen.

Structures with more than two neighboring RADaBs are vacancy-like point defects and are very unstable. Such partial vacancies, along with interstitial $\mathrm{H}_{3}$ site atoms, are responsible for structural changes on $\mathrm{Ge}(111)$. The rate at which they propagate can be estimated from our STM images. The configuration with an interstitial $\mathrm{H}_{3}$ site $\mathrm{Ge}$ adatom must have a lifetime of seconds or only slightly less to be visible in STM images taken at 70 degrees Celsius Figure 6 . Thus, we estimate that the activation energy for the propagation of an interstitial $\left.\mathrm{H}_{3}{ }_{3}\right)$ site along the row is around $0.8 \mathrm{eV}$. The lifetime of a partial vacancy must be even shorter because our scan speed is too slow to detect its propagation. The activation energy for the propagation of a partial vacancy must therefore be less than $0.65 \mathrm{eV}$.

The creation of these two mobile point defects is thermally activated. Within a domain of the 2 times row structure, partial vacancies and interstitial $\mathrm{H}_{3}$ site atoms are formed in pairs. To create a partial vacancy-interstitial pair within a row, an atom must jump to an $\mathrm{H}_{3}$ site and its neighbor must be available to trap it there, by the mechanism illustrated for $\mathrm{a} \mathrm{Pb}$ atom in Figure 4B. We can estimate the formation energy of the pair in a perfect region of $\mathrm{Ge}(111)-\mathrm{c}(2$ times 8$)$. The mean lifetime for a Ge adatom to stay at the $T_{4}$ site is (from Equation land the discussion preceding it) $10^{\text {negative }} 13 \exp \left(0.8\right.$ over $\left.k_{B} T\right) \mathrm{s}$ and its lifetime on the $\mathrm{H}_{3}$ site is $10^{\text {negative }} 13 \exp \left(0.2\right.$ over $\left.\mathrm{k}_{\mathrm{B}} \mathrm{T}\right) \mathrm{s}$. The generation rate for the partial vacancy-interstitial pair is given by the product of three terms: (i) the rate at which an atom jumps to an $\mathrm{H}_{3}$ site, (ii) the probability that a neighboring atom is on an $\mathrm{H}_{3}$ site and thus available to trap the first atom, and (iii) a geometrical factor of order 0.1 because there are at least two $\mathrm{H}_{3}$ sites available for each jump. The rate at which an atom jumps to an $\mathrm{H}_{3}$ site is the inverse of its lifetime on the $T_{4}$ site, $10^{13} \exp$ (negative 0.8 over $k_{B} T$ ) $s^{\text {negative }} 1$. The probability that another atom will be on an $\mathrm{H}_{3}$ site is the ratio of its lifetime on the $\mathrm{H}_{3}$ site to that on the $\mathrm{T}_{4}$ site, which is exp(negative 0.6 over $k_{B} T$ ). Therefore, the interstitial-vacancy pair generation rate is about $10^{12}$ $\exp$ (negative 1.4 over $\mathrm{k}_{\mathrm{B}} \mathrm{T}$ ) $\mathrm{s}^{\text {negative }} 1$ per atom. Thus, the formation energy for the pair in perfect $\mathrm{c}(2$ times 8$)$ is similar to $1.4 \mathrm{eV}$ [21].

Because perfect $\mathrm{c}(2$ times 8$)$ is the ground state structure on $\mathrm{Ge}(111)$, the formation energy of mobile defects will be lower at domain boundaries and in other regions of the surface that are not in a perfect 
c(2 times 8) configuration. For example, we observe shifts much more frequently in adatom rows next to an extra row (such as the one indicated by an arrow in Figure 1 than in a perfect $\mathrm{c}(2$ times 8$)$ domain. Likewise, more atomic motions occur near domain boundaries. This assumption is reasonable, based on our discussion of the stability of various structures. Because the configuration at the domain boundaries is a less favorable one than the $2 x$ row structures, the barrier for an adatom there to hop to $\mathrm{an}_{\mathrm{H}}$ site is less than that for an adatom in perfect $\mathrm{c}(2$ times 8$)$. Thus, we expect the activation energy for creation of mobile point defects at domain boundaries will be less than $1.4 \mathrm{eV}$, and adatom motion will occur at boundaries at a lower temperature than it will inside large domains.

\section{Phase Transition of Ge(111)-c(2 times 8) double head arrow 1 times 1 at similar to 300 degrees Celsius \pm}

The creation and propagation of partial vacancies and interstitial $\mathrm{H}_{3}$ site atoms may lead to the $\mathrm{c}(2$ times 8 ) double head arrow ( 1 times 1 ) phase transition on $\mathrm{Ge}(111)$. This transition has been studied by a number of techniques, but the detailed mechanism responsible for the formation of the high-temperature 1 times 1 phase is still not well understood. Phaneuf and Webb did a detailed study of the phase transition using LEED [22]. They observed that above the transition temperature (similar to 300 degrees Celsius), the eighth-order and fourth-order peaks disappeared and half-order peaks broadened, split, and decreased in intensity with increasing temperature. The average domain size decreased from about 37 to about 17 angstrom as the temperature increased from 300 degrees to 600 degrees Celsius [22]. A later He diffraction study confirmed many of these observations [23]. A core-level study of the phase transition by Aarts et al. indicated that no significant changes occur in either the binding energies or in the relative intensities of the two surface components [17], which implies that the ordered adatom structure becomes disordered without an appreciable change in the number of adatoms present or in the nature of their binding to the first-layer atoms. Photoelectron diffraction, ellipsometry, and LEED studies also showed that the local adatom structure was unchanged during the phase transition [22,24,25]. A recent direct STM observation by Feenstra et al. showed that the transition is caused by adatom diffusion [7]. However, the atomic motions in the disordered 1 times 1 phase are very fast, and thus, the detailed structure in the 1 times I phase could not be resolved. The disordered region started from the domain boundaries of $c$ ( 2 times 8 ) 
at similar to 200 degrees Celsius and grew continuously with the temperature until the entire surface became disordered at 300 degrees Celsius. They also observed that some of the adatom rows in the $c(2$ times 8) reconstruction become "fuzzy." We also studied the clean $\mathrm{Ge}(111)$ surface up to similar to 200 degrees Celsius. We observed rapid adatom motions at domain boundaries and fuzzy rows, consistent with their results [7]. We also occasionally observed the nucleation of a region of rotated row structures.

All of these results can be explained in terms of the creation and propagation of mobile point defects on the $\mathrm{Ge}(111)-\mathrm{c}(2$ times 8 ) surface and the row shifts and the nucleation of rotated domains of $\mathrm{c}(2$ times 8) to which they lead. As the temperature increases, more of the mobile point defects are created. In the last section, we calculated the formation energy for the interstitial site-vacancy pair in perfect $c(2$ times 8 ) to be $1.4 \mathrm{eV}$. The generation rate is then estimated to be on the order of 10 (negative) $13,10^{\text {negative }} 7,10^{\text {negative }} 3$, and $1 \mathrm{~s}^{\text {negative }} 1$ per atom at 25 degrees, 100 degrees, 200 degrees, and 300 degrees Celsius, respectively. These estimates indicate that the effects of $\mathrm{Ge}$ adatom motions can be observed near 200 degrees Celsius and that at 300 degrees Celsius they would be too fast for the STM to image, which agrees well with experimental observations [7]. Because of the much higher propagation rate of the partial vacancy compared with that of an interstitial $\mathrm{H}_{3}$ site, it is likely to be the dominant cause for the structural changes occurring on this surface.

We therefore propose that the surface structure at high temperature (greater than 300 degrees Celsius) is composed of small domains (tens of angstroms in diameter) of the $2 x$ row structures, which have row directions in any one of the three possible $<01$ bar $1>$ directions. Inside the domains, there are adatom row shifts occurring as a result of the propagation of the two types of mobile point defects that are mainly created at domain boundaries, consistent with STM results [7]. At a fixed temperature, one domain can grow at the expense of another, but the average size remains constant. As the temperature is increased, the excitation that forms a new domain of rotated row structure becomes more probable, and the average size of the domains shrinks, as observed in LEED [22]. As the ordered c(2 times 8) regions disappear at similar to 300 degrees Celsius, the $8 x$ and $4 x$ periodicity is lost, but the $2 x$ periodicity of the row structure is still present, in agreement with LEED and He diffraction [22,23].

The structure of a possible configuration in the high-temperature 1 times 1 phase Figure 10 is somewhat disordered and composed of 
domains of the row structure. Between domains there are local structures of square root of 3 times square root of 3 and of two neighboring RADaBs. There are also local structures with an adatom trapped on the interstitial $\mathrm{H}_{3}$ site and with a high density of RADaBs, and they become more abundant with increasing temperature. The 2 times 2 has threefold symmetry, and its row direction can be assigned to any one of the three possible $<01$ bar $1>$ directions. The incommensurate structure proposed by Phaneuf and Webb, which is composed of domains of 2 times 2 separated by walls in the c ( 2 times 4) local arrangement [22], is a special case of this model. Therefore, the main features of the diffraction pattern can be expected to be similar for both models. However, our model additionally explains the growth of the disordered region from domain boundaries between 200 degrees to 300 degrees Celsius, the change in average domain size with increasing temperature, and the "fuzzy" rows. Furthermore, it provides a mechanism, the motion of mobile point defects, for the transition from the $\mathrm{c}(2$ times 8$)$ reconstruction to the hightemperature phase.

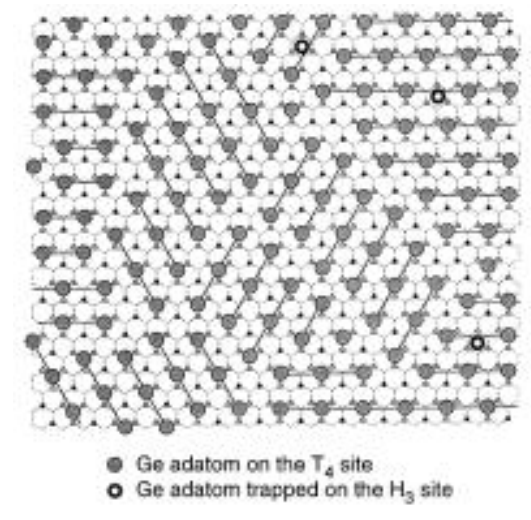

Figure 10. Model of a snapshot of a possible configuration of the high-temperature 1 times 1 phase. The row direction of each domain is marked.

[Help with image viewing]

[Email Jumpstart To Image]

Feenstra et al. observed that the disordered region forms a band with a fixed average width between two translationally inequivalent domains of $\mathrm{c}(2$ times 8$)$, which is in equilibrium with the ordered domains [7]. We believe that the surface stress caused by large domains of the $\mathrm{c}(2$ times 8 ) reconstruction is responsible for the stabilization of the disordered region below 300 degrees Celsius and for the continuous growth of the disordered region from 200 degrees 
to 300 degrees Celsius. Spectroscopic ellipsometry by Abraham et al. suggested that the surface stress plays a role in the phase transition [24]. An important role for surface stress in this transition would be consistent with observations of phase transitions on other semiconductor surfaces, such as the $\mathrm{Si}(111)-7$ times 7 to 1 times 1 transition at similar to 860 degrees Celsius and the Pb over Ge(111) square root of 3 times square root of 3 to 1 times 1 transition at similar to 180 degrees Celsius. In all three transitions, the disordered regions grow continuously with the temperature from a boundary: domain boundaries in the Ge(111) system, step edges on Si(111) $[8,26]$, and phase boundaries in Pb over Ge(111) [6]. The bonding in semiconductors is strong and directional, and thus, a large domain of a surface reconstruction can generate significant stress in the underlying substrate. The stress can be expected to dramatically change the surface energetics at step edges, domain boundaries, or phase boundaries. Therefore, great care should be taken in the analysis of phase transitions on semiconductor surfaces when nonmicroscopic techniques are used.

\section{Conclusion ㄴ}

We have presented a microscopic mechanism for adatom motions on $\mathrm{Ge}(111)$. The fundamental units responsible for these motions are the two complementary types of mobile point defects: an interstitial $\mathrm{H}_{3}$ site $\mathrm{Ge}$ adatom and a partial vacancy. The $\mathrm{Ge}(111)-\mathrm{c}(2$ times 8$)$ double head arrow ( 1 times 1 ) phase transition is explained by the generation and propagation of these two types of mobile point defects. Thus, very complex and beautiful transformations can arise from the motions of two fundamental point defects.

\section{REFERENCES AND NOTES최}

1. B. S. Bokshtein, S. Z. Bokshtein, A. A. Zhukhovitskii, Thermodynamics and Kinetics of Diffusion in Solids (American Publishing, New Delhi, India, 1985). [Context Link]

2. W. Frank, U. Goesele, H. Mehrer, A. Seeger, in Diffusion in Crystalline Solids, G. E. Murch and A. S. Nowick, Eds. (Academic Press, London, 1984), chap. 2. [Context Link]

3. M. G. Lagally, Phys. Today 46, 24 (November 1993), and references therein. [Context Link]

4. E. Ganz, S. K. Theiss, I.-S. Hwang, J. Golovchenko, Phys. Rev. Lett. 68, 1567 (1992). [Context Link] 
5. I.-S. Hwang and J. Golovchenko, Science 258, 1119 (1992). [Context Link]

6. , Phys. Rev. Lett. 71, 255 (1993). [Context Link]

7. R. M. Feenstra et al., ibid. 66, 3257 (1991). [Context Link]

8. S. Kitamura et al., Nature 351, 215 (1991). [Context Link]

9. M. Hansen, Constitution of Binary Alloys (McGraw-Hill, New York, 1958),

p. 771. [Context Link]

10. J. J. Metois and G. Le Lay, Surf. Sci. 113, 422 (1983). [Context Link]

11. R. S. Backer, J. A. Golovchenko, B. S. Swartzentruber, Phys. Rev. Lett. 54, 2678 (1985). [Context Link]

12. R. Feidenhans'l et al., Phys. Rev. B 38, 9715 (1988). [Context Link]

13. R. S. Becker, B. S. Swartzentruber, J. S. Vickers, T. Klitsner, ibid. 39, 1633 (1989). [Context Link]

14. T. Klitsner and J. S. Nelson, Phys. Rev. Lett. 67, 3800 (1991). [Context Link]

15. N. Takeuchi et al., ibid. 69, 648 (1992). [Context Link]

16. R. M. Feenstra and A. J. Slavin, Surf. Sci. 251-252, 401 (1991). [Context Link]

17. J. Aarts, A.-J. Hoeven, P. K. Larsen, Phys. Rev. B 38, 3925 (1988). [Context Link]

18. N. Takeuchi et al., ibid. 49, 10757 (1994). [Context Link]

19. The energy of a Ge-Ge bond is $1.6 \mathrm{eV}$ in the bulk [27]. A jump involving the breaking of two Ge-Ge bonds would presumably have an activation energy greater than $1.6 \mathrm{eV}$. It would be less than 3. $2 \mathrm{eV}$, however, because of lattice relaxation and charge transfer during the movement. [Context Link]

20. T. Klitsner, R. S. Becker, J. S. Vickers, Phys. Rev. B 44, 1817 (1991). [Context Link]

21. This activation energy will be less on $\mathrm{Pb}$-doped surfaces, because $\mathrm{Pb}$ jumps more readily to $\mathrm{H}_{3}$ and has a longer lifetime there, so it can be readily trapped. Additionally, long jumps of $\mathrm{Pb}$ atoms can leave behind a vacancy in one row and create an interstitial defect in another. [Context Link]

22. R.J. Phaneuf and M. B. Webb, Surf. Sci. 164, 167 (1985). [Context Link] 
23. J. S. Ha and E. F. Greene, J. Chem. Phys. 91, 7957 (1989). [Context Link]

24. M. Abraham, G. Le Lay, J. Hila, Phys. Rev. B 41, 9828 (1990). [Context Link]

25. L. Patthey, E. L. Bullock, K. Hricovini, Surf. Sci. 269-270, 28 (1992). [Context Link]

26. N. Osakabe, Y. Tanishiro. K. Yagi, G. Honjo, ibid. 109, 353 (1981). [Context Link]

27. C. Kittel, Introduction to Solid State Physics (Wiley, New York, ed. 5, 1976), p. 94. [Context Link]

28. We acknowledge helpful discussions and criticism of the manuscript by $\mathrm{E}$. Kaxiras and S. D. Theiss. Supported by the Materials Research Laboratory at Harvard University (contract NSF-DMF-8920490) and the Joint Services Electronics Project (contract N00014-89-J-1023).

Accession Number: 00007529-199407220-00003

Copyright $(1) 1994$ by the American Association for the Advancement of Science Volume 265(5171), 22 July 1994, pp 490-496 